\title{
Plasma Modification of 3D Hydrogels for Cell Culture Applications
}

\author{
Emel Sokullu \\ İzmir Katip Çelebi University, Faculty of Engineering Sciences \\ Bioengineering Department, Harmandalı, Çiğli, İzmir, Turkey \\ emelsu@gmail.com \\ Recieved: $30^{\text {th }}$ November 2016 \\ Accepted: $3^{\text {rd }}$ February 2017 \\ DOI: http://dx.doi.org/10.18466/cbujos.302649
}

\begin{abstract}
The aim of this study was to examine plasma effect on to the different structures including 2D glass slides and 3D treated Gelma hydrogels with an increased range of biodegradation rates for more rapid in vivo biodegradation in tissue engineering and bioactive factor delivery applications. Considering the known excellent biocompatibility of GELMA, we envision that this hydrogel material could be potentially used in tissue engineering and for the purpose of directing the growth and migration of cells. Another dimension of this study is plasma patterning of the substrates. This method has been employed to form diverse networks of different cell types for investigations involving migration, signaling, tissue formation, and the behavior and interactions of neurons arraigned in a network. As a result of these, we investigated whether the swelling behavior, degradation profiles, and cell proliferation properties of photocrosslinked hydrogels formed by methacrylated and plasma treated are tunable by varying the degree of plasma conditions. The potential applicability of these photocrosslinked GELMA hydrogels as a carrier of embryonic stem cells (ESCs) was also examined in vitro.
\end{abstract}

Keywords - Plasma treatment, hydrogels, 3D Cell Culture, embryonic stem cells (ESCs) .

\section{Introduction}

Tissue engineering (TE) using biomaterial scaffolds offers a promising alternative to enhance the efficiency of cell transplantation, and the use of biomaterial to deliver cells into the injured tissue might increase cell survival. Hydrogel is one of the most commonly used scaffolds for cell delivery and tissue regeneration because of its highly hydrated three-dimensional (3D) polymeric networks which are structurally similar to the extracellular matrix (ECM), providing sites for adhesion, proliferation and even differentiation of cells [1]. Gelatin-based hydrogels have good biocompatibility and low cost. They have also been approved by the US Food and Drug Administration (FDA) as cell delivery vehicles and hemostatic agents [2]. However, they lack a sufficient number of energy dissipation mechanisms to slow crack propagation with irregularly distributed cross-linking points [4]. In literature, various techniques such as the incorporation of polymer nanoparticles [4-6], inorganic clay [7] and carbon nanotubes [8] or interpenetrating polymer network or double-network structure formation $[9,10]$ have been attempted with the aim of improving the properties of hydrogels.

In general, the crosslinked structure of hydrogels is characterized by junctions or tie points, which may be formed from strong chemical linkages (such as covalent and ionic bonds), permanent or temporary physical entanglements, microcrystallite formation, and weak interactions (such as hydrogen bonds) $[1,11]$. For cross-linking and network formation, 
several options for preparation have been developed. Ultimate network morphology of a hydrogel can be amorphous, semicrystalline, H-bonded, supramolecular, or consisting of hydrocolloidal aggregates [1,11]. The chains comprising the network may be based on natural, synthetic, or hybrid combinations of these materials. The physical structure and characteristics of hydrogels depend upon starting monomers and macromers, better cellular attachment compared to uncharged gels. Furthermore, more hydrogels used in regenerative medicine are being synthesized from natural macromers, which are typically ionic or ionizable. As this trend continues, consideration must be given to the inherent differences in solute transport and cell and protein adherence compared to neutral gels [1, 11,12].

Effective solute transport is one of the most critical design parameters for hydrogels in regenerative medicine. Mass transport parameters determine how nutrients, gasses, waste products, and bioactive agents, such as growth factors to stimulate natural tissue growth[1,13] are exchanged within scaffolds or are delivered by the gel. Except in hydrogels with very large micropores or forced flow conditions, convection usually does not play a significant role in the movement of solutes through hydrogel matrices. Diffusion alone is regarded as the driving transport phenomenon. Analysis of drug and protein diffusion by Ende et al.[1,14] in ionic gels revealed that mesh size and environmental conditions, including $\mathrm{pH}$ and temperature, are all critically important in solute diffusion. They further concluded that hydrogels might be tailormade for the release of a specific drug, protein, or peptide. Other studies showing the effect of $\mathrm{pH}$ on drug transport from ionized hydrogels done by Brannon- Peppas and Peppas [1,15] showed that $\mathrm{pH}$-dependent hydrogels could be prepared to exhibit no release, an important property for drug delivery systems.

With biological systems, where both the polymer and the solute are frequently ionized, the interactions between the polymer and solute themselves become an important factor in determining transport behavior Collins and Ramirez [16] stud- ied. Since both equilibrium swelling theory and rubber elasticity theory rely on experimentally determined gel properties to determine average molecular weight between crosslinks, these models are useful for noncovalently crosslinked hydrogels as well. Hydrogels used in regenerative medicine may be formed by physical entanglements, microcrystallites, or hydrogen-bonded structures. In a general way, these theories may be extended to systems of this type by treating the junctions or tie points that cause gel formation as physical crosslinks with equivalent behavior as the covalent crosslinks intended with the above theories.

Hydrophilic polymers can swell and absorb water without dissolving, provided that chemical or physical crosslinks exist among the macromolecular chains. The polymer network resulting from the crosslinks swells in the aqueous solvent, until the thermodynamic force of swelling is totally counterbalanced by the elastic, retractive force exerted by the crosslinks. This 'solid-like solution' of polymer and water resulting at equilibrium is known as a hydrogel. The amount of water retained by the mesh of the hydrogel network depends on the structure of the polymer network itself and on the environmental conditions, such as the temperature, $\mathrm{pH}$ and ionic strength of the water solution in contact with the polymer $[17,18]$. The volume or mass swelling ratio of the hydrogel is the most important variable to be evaluated for given environmental conditions, as it affects the diffusive, mechanical, optical, acoustic and surface properties of the hydrogel itself. In cases where sharp and/or fast swelling-deswelling transitions happen in response to changes of external stimuli, hydrogels are potentially useful for the development of a variety of smart devices, such as valves, artificial muscles and substrates for controlled drug release [18-22].

In these regards, plasma processing of hydrogels has been evaluated. Plasma effect on 2D surfaces already reported in literature these effects on 3D hydrogels structure is rare. Although plasma is mainly effected on to the surface, it is expected chain reactions would be extended inside as well. Because of the surface characteristics also greatly influence attachment and growth of cells on bio- 
materials, surface performance of plasma-treated hydrogels can be studied with cells. With this aim in the literature, argon plasma treated PEGDAHEMA hydrogel morphologically and chemically examined [23]. The contact angle to water decreased considerably after argon plasma treatment compared to the untreated samples was caused by the changes in morphology (SEM images) and chemical composition (XPS results) of the argon plasma-treated surface. In that study, the increase of $\mathrm{O} / \mathrm{C}$ ratio confirmed that the oxygen-containing groups were incorporated onto the PEGDA-HEMA film surface, which led to the improvement of surface hydrophilicty of the films. As morphology changes could cause hydrophilicity of the films changes, the surface free energy was increased for the argon plasma- treated films compared to that of the untreated. The prepared samples were kept in air after plasma treatment; plasma oxidation reaction produced oxygen-containing functional groups, which were attached to the polymer surface. These functional groups formed and played an important role in increasing the hydrophilic properties of the hydrogel. The results indicated that surface free energy of the hydrogel films increased with increasing the plasma treatment power and time, mainly due to the increase of polar component.

Oxidized gels are important and were synthesized to accelerate the degradation rate of several gels [24-32]. This approach offers control over the degradation rate by varying the oxidation degree, as increasing the oxidation degree can increase the vulnerability of hydrogels to hydrolysis [26] and [28]. Therefore, we used plasma oxidized to argue that to produce open-chain, which is more intended to hydrolytic degradation [28]. In this manner, hydrolytic degradation could take place both at the gelatin polymer backbone and at the ester bonds in the photopolymerized crosslinks [30].

Additionally, systematic manipulation of a cell microenvironment with micro- and nanoscale resolution is often required for deciphering various cellular and molecular phenomena. To address this requirement, in this study, we also have developed a plasma lithography technique to manipulate the cellular microenvironment by creating a patterned surface with feature sizes ranging from $150 \mathrm{um}$. to millimeters. The goal of this technique is to be able to study, in a controlled way, the behaviors of individual cells as well as groups of cells and their interactions [31,32]. This plasma lithography method is based on selective modification of the surface chemistry on a substrate by means of shielding the contact of low-temperature plasma with a physical mold. This selective shielding leaves a chemical pattern which can guide cell attachment and movement. This pattern, or surface template, can then be used to create networks of cells whose structure can mimic that found in nature and produces a controllable environment for experimental investigations. The technique is well suited to studying biological phenomenon as it produces stable surface patterns on transparent polymeric substrates in a biocompatible manner. The surface patterns last for weeks to months and can thus guide interaction with cells for long time periods which facilitates the study of long-term cellular processes, such as differentiation and adaption. The modification to the surface is primarily chemical in nature and thus does not introduce topographical or physical interference for interpretation of results. It also does not involve any harsh or toxic substances to achieve patterning and is compatible for tissue culture. Furthermore, it can be applied to modify various types of polymeric substrates, which due to the ability to tune their properties are ideal for and are widely used in biological applications. The resolution achievable is also beneficial, as isolation of specific processes such as migration, adhesion, or binding allows for discrete, clear observations at the single to multicell level.

In this study, we evaluated plasma effect on 3D hydrogel tissue elements in order to swelling characteristics and biodegradation dynamics together with the in vitro biocompatibility of the scaffolds. We also evaluated plasma effect of ESCs viability in 3D culture environment. 


\section{Material and Methods}

\subsection{Materials and hydrogel precursor preparation}

In this study, Gelatin (Type A, 300 bloom from porcine skin), methacrylic anhydride (MA) and 3(trimethoxysilyl) propyl methacrylate (TMSPMA) were purchased from Sigma-Aldrich (St. Louis, MO). Photoinitiator 2-hydroxy-1-[4(hydroxyethoxy)-phenyl]-2-methyl-lpropanone (Irgacure 2959) was purchased from Ciba Geigy (Igracure $^{\mathrm{TM}}$ 2959, Ciba Speciality Chemicals, Tarrytown, NY, USA). The live/dead viability/cytotoxicity kit for mammalian cells was purchased from Invitrogen Corporation (Molecular Probes, Invitrogen, Carlsbad, USA). Glass slides and coverslips (VWR VistaVision ${ }^{\mathrm{TM}}$ microscope cover glasses, $25 \times 25 \mathrm{~mm}$ ) were purchased from Fisher Scientific (Philadelphia, USA). The Omnicure $^{\circledR}$ S2000 UV/Visible Spot Curing System from EXPO Photonic Inc. (Ontario, CANADA) was used to polymerize hydrogels.

\subsection{Synthesis of gelatin methacrylate precursor}

Gelatin methacrylate (GelMA) was synthesized by dissolving $10 \%(\mathrm{w} / \mathrm{v})$ gelatin (Type A, 300 bloom from porcine skin) into Dulbecco's phosphate buffered saline (DPBS; GIBCO) at $45-50{ }^{\circ} \mathrm{C}$ for 1 hour. Methacrylic anhydride (94\%, MA, Sigma-Aldrich, St. Louis, MO)) was added to the solution to crosslink with gelatin at $50{ }^{\circ} \mathrm{C}$ under stirring condition for 2 hours. This solution was dialyzed in distilled water using a 12-14 kDa dialysis bag (Fisher Scientific, Philadelphia, USA) for 1 week at $40{ }^{\circ} \mathrm{C}$. The dialyzed solution was then transferred into $50 \mathrm{~mL}$ centrifuge tube and lyophilized for one week to generate usable GelMA powder. GelMA precursor solution was prepared by mixing $5 \%(\mathrm{wt} / \mathrm{wt})$ gelatin methacrylate and 1\% (wt/wt) 2-hydroxy-1-(4(hydroxyethoxy)phenyl)-2-methyl-1-propanone photo-initiating powder (Igracure ${ }^{\mathrm{TM}}$ 2959, Ciba Speciality Chemicals, Tarrytown, NY, USA) in DPBS.

\subsection{Plasma Processing}

$5 \mathrm{ml}$. prepolymer solution was poured into $6 \mathrm{~cm}$. petri-dishes and exposed to $6.9 \mathrm{~mW} / \mathrm{cm} 2 \mathrm{UV}$ light (360-480 nm) for 90s. Samples were detached from the slide and incubated at $37^{\circ} \mathrm{C}$. Then the samples has been put into Plasma Chamber (Harrick Plasma, USA) and processed with low, medium and high RF levels. For this aim, air plasma - PDC-32G Harrick plasma equipped with a dry vacuum pump has been utilized in Low $-10 \mathrm{~mA}, 6.8 \mathrm{~W}$, Medium $-15 \mathrm{~mA}, 10.5 \mathrm{~W}$ and High $-25 \mathrm{~mA}, 18 \mathrm{~W}$ dose energy values.The base pressure was $8 \mathrm{~Pa}(60$ mTorr). The operating pressure flow during experiments is $67 \mathrm{~Pa}$ (500 mTorr). Process time was 30 sec.-2min.

\subsection{PDMS mold preparation for Plasma Lithogra- phy}

The desired pattern is designed and then sent to an outside company for producing a photomask. Photolithography method has been used to produce master pattern. Glass slides are patterned with either a thin positive resist or a thick negative resist depending upon the size of the structure being created. Glass slides are used as substrate. The patterned slide is attached to a stack of slides which have not been patterned. A container of tinfoil slightly larger than the stack of slides is created to hold the 30g. of Polydimethylsiloxane (PDMS) which is then poured over the photolithographically patterned surface to create an initial mold. The PDMS is degassed and allowed to cure for one to two days. Once cured the PDMS is peeled off of the master pattern and forms a mold for the next step. $6 \mathrm{~g}$. of 2 part epoxy ( Devcon \#14310(6 g) is mixed for 1 minute and then poured into the master mold, degassed and allowed to cure. The 6 g. used produces a thin layer which facilitates quick bubble removal. The epoxy is then allowed to cure for one to two days. A working mold is then created by pouring PDMS onto the epoxy mold, degassing the PDMS, and curing for one to two days. Once cured, the working mold is peeled off of the epoxy and stored to maintain cleanliness. 


\subsection{Swelling and Dehydration}

Polymerization was performed as described above. Immediately following hydrogel formation, $8 \mathrm{~mm}$ radius disc of each composition was punched from a flat thin sheet and placed in DPBS at $37^{\circ} \mathrm{C}$ for 24 $h$. Discs were removed from DPBS and blotted with a KimWipe to remove the residual liquid and the swollen weight was recorded. Samples were then lyophilized and weighted once more to determine the dry weight of polymer. The mass swelling ratio was then calculated as the ratio of swollen hydrogel mass to the mass of dry polymer. The gels were then incubated in DPBS at room temperature for 48 $\mathrm{h}$ more, weighted, and the rehydrated ratio was calculated as the ratio of the rehydrated wet mass to the initial wet mass. The sample size for analysis was six gels per group.

\subsection{Biodegradation}

Polymerization was performed as described above. Hydrogels were placed in $1.5 \mathrm{~mL}$ tubes with $1 \mathrm{~mL}$ of DPBS with $2.5 \mathrm{U} / \mathrm{mL}$ of collagenase type II (Worthington Biochemical). Gels were incubated with collagenase type II at $37^{\circ} \mathrm{C}$ for $3,6,12,24,36$, or $48 \mathrm{~h}$. After each time point, the collagenase solution was removed, without disturbing the undigested hydrogel. The remaining hydrogel was washed with DPBS, and then all liquid was removed and gels were lyophilized. The percentage of degradation was calculated by the dried weight after digestion divided by the weight of untreated hydrogels. The sample size was six gels per group.

\subsection{Cell encapsulating and testing cell viability}

ESCs were cultured in Glasgow Minimum Essential Medium (Sigma Aldrich, USA) supplemented with 15\% Fetal bovine serum (Invitrogen, Carlsbad, CA, USA) and 1\% Penicillin Streptomycin (Invitrogen, Carlsbad, CA, USA) $1 \%$ Sodium Pyruvate (Invitrogen, Carlsbad, CA, USA) , 1\% Non-essential amino acids (NEAA) (Invitrogen, Carlsbad, CA, USA), $0.1 \%$ B-Mercaptoethanol (Invitrogen, Carlsbad, CA, USA) and 0.01\% Leukemia Inhibitory Factor (Millipore Darmstadt, Germany). Cell viability was tested using a live/dead assay (Molecular
Probes, Invitrogen, Carlsbad, CA, USA) at day 1,4 and 7. Tissue prototypes were first washed with DPBS and gels were incubated with $0.5 \mu \mathrm{l} / \mathrm{ml}$ of calcein-AM (for live cells) and $2 \mu \mathrm{l} / \mathrm{ml}$ of ethidium bromide (for dead cells) in DPBS. The fluorescent images were taken using an inverted microscope (Nikon TE2000). Cell viability was calculated as the average value of the fraction of live cells to the total number of cells taken from 5 samples. Viability of encapsulated ESCs $\left(5 \times 10^{5}\right.$ cells $/ \mathrm{ml}$.), was quantified in fluorescent microscope images using the $\mathrm{NIH}$ Image program (developed at the U.S. National Institutes of Health and available at http://rsb.info.nih.gov/nih-image/). Cell culture medium was changed daily.

\section{Results and Discussions}

We applied plasma on UV crosslinked hydrogels and evaluated the swelling characteristics and biodegradation kinetics of low, medium and high RF plasma treated and untreated micro-hydrogels. This is important in various applications as it affects solute diffusion, surface properties, mechanical properties, and surface mobility. The degree of swelling of gels is dependent on the pore size of the polymer network and the interaction between the polymer and the solvent. Hydrogels were made as described previously high, low and medium RF plasma treated. They were allowed to reach the equilibrium over a $24 \mathrm{~h}$. incubation in DPBS at room temperature, then the mass swelling ratio of the swollen mass to the dry mass of polymer was calculated and compared (Figure 1.). As can be seen in Figure 1., by exposing the RF plasma, the mass swelling ratio increased significantly with decreasing RF plasma treatment at constant hydrogel concentrations, demonstrating that the RF plasma exposure had a significant effect on the material's ability, and aptitude for attracting and storing water within the polymer network. As swelling can have a profound effect on the overall shape of patterned hydrogels, especially when micro/nano patterned in craggy shapes. This data suggests that pattern accuracy would be improved by increasing the RF degree. 


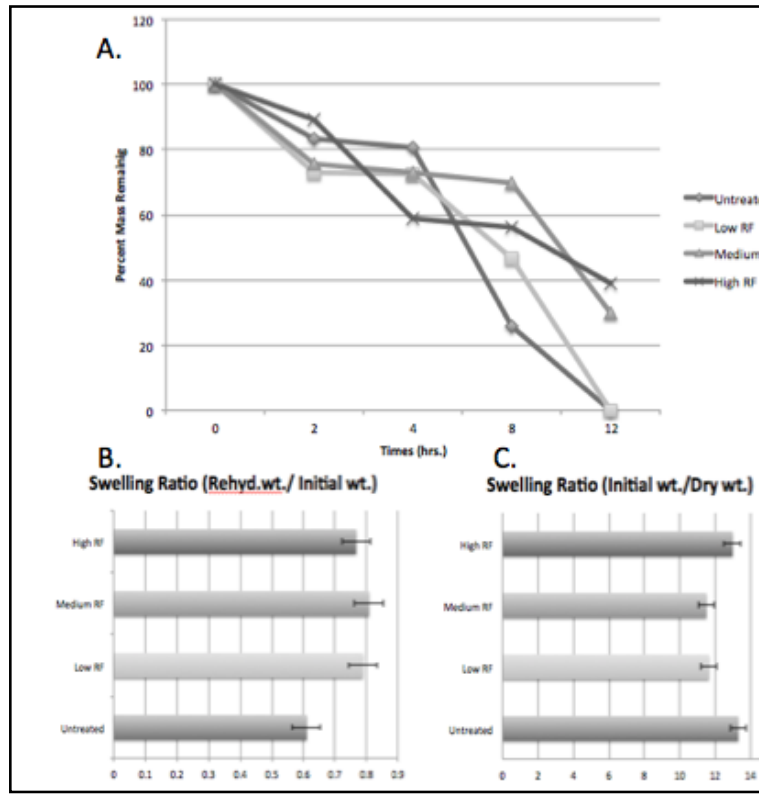

Figure 1. Biodegradation Behavior of Untreated, Low, Medium and High RF Plasma Treated Samples (A.) and Their Swelling Ratio Comparisons by Rehydrated Weight/ Initial Weight (B.) and Initial Weight / Dry Weight (C.).

Figure 2. shows that, ESC cell viability comparison of 3D Untreated (Control) and 20, 60, 90,120 sec. plasma treated samples. Here it can be seen that cell viability increasing with lower exposure to RF plasma treatment on the other hand while the exposure time increasing, cell viability tended to decreasing. In order to long term culture results, which can be shown in Figure 2B. although cell viability is decreasing just after the plasma exposure (1st. Day), by extending the culturing period $\left(4^{\text {th }}\right.$. And $7^{\text {th }}$. Days) it tends to increasing. This can be explained by stress of the cells is effecting its proliferation and recovering of vacuum effect might positively effecting on cell proliferation. Below in Figure 3., it also can be seen that cell clusters are formed less on plasma treated samples in compare with the untreated ones.
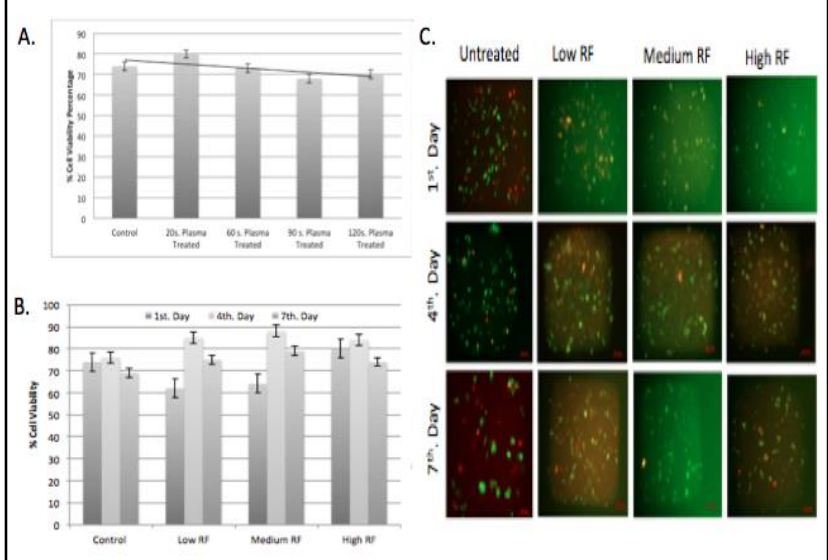

Figure 2. ESC Cell Viability Comparison of 3D Untreated (Control) and 20, 60, 90,120sec. Plasma Treated Samples (A.) Dose of Plasma Comparison Through Untreated (control), Low, Medium and High RF Plasma Treated in Constant Time (20s.) for GELMA Microgels (B.) and Their Live/Dead Stained Phase Contrast images (C.)

Plasma lithography experiment results can be seen on Figure 4. In this experiment we have used photomasks with the sizes of $1000 \times 1000 \mathrm{~mm}$.-A.B. and 150x150 $\mu \mathrm{m}-$ C.D. As a result of this, we achieved that the plasma lithographically patterned surfaces and fibroblast cells are tended to attach on plasma treated regions. Live/Dead assay results are proved that fibroblast cells on patterned regions are creating living cell lines on to the surface (Figure 4.).

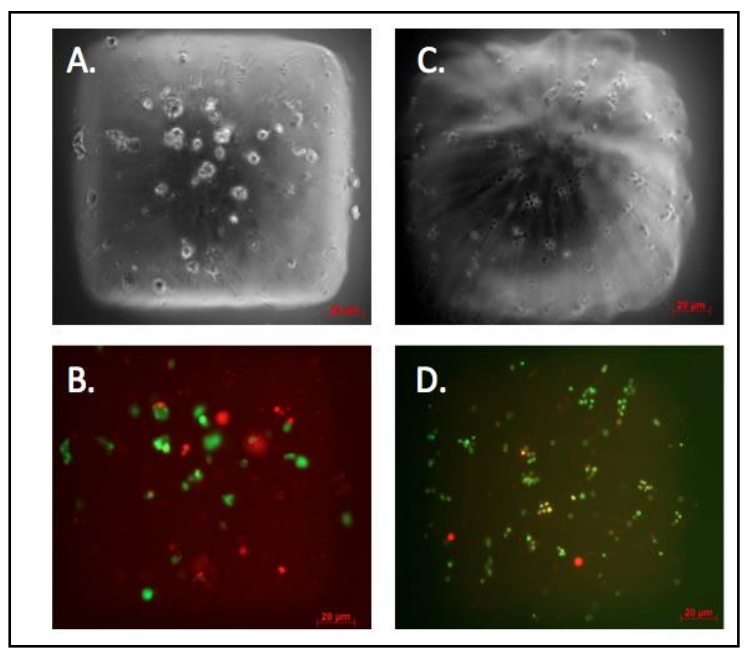

Figure 3. Live/Dead Cell Viability Results of Untreated (A., B.) and Plasma Treated (C., D.) 3D GELMA Microgel Samples. 

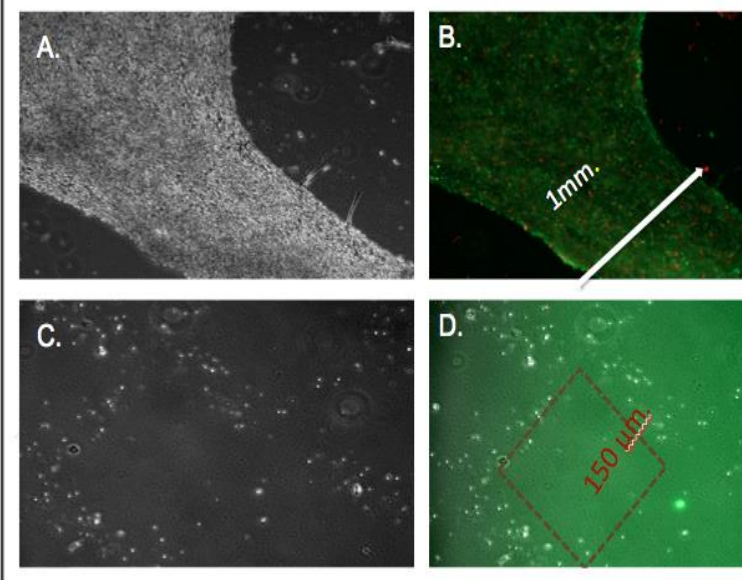

Figure 4. Live/Dead Cell Viability Results of 3T3 Fibroblasts, Patterned by Plasma Lithography Techniques (Mask sizes are 1000x1000mm.A.B. and 150x150 $\mu \mathrm{m}-$ C.D.).

\section{Conclusion}

In this work, we have evaluated swelling and biodegradation properties of plasma treated 3D GELMA hydrogels. We also demonstrated the fabrication of embryonic stem cell encapsulating microgels and we applied plasma treatment on them. We evaluated the compatibility of cell encapsulating microgel fabrication technology with ESCs by performing compatibility and viability assays in vitro. Encapsulated and plasma treated ESCs presented here offers new opportunities as a platform technology for $3 \mathrm{D}$ in vitro studies. Further, plasma treatment of micron sized gels enables broad clinical applications for engineering tissue systems. As the organotypic 3D model mimics human tissues, it opens up new experimental approaches to explore the effect of radiation in vivo and will have important implications for evaluating radiation risk on human tissue carcinogenesis and cancer therapy.

\section{Acknowledgement}

The Scientific and Technical Research Council of Turkey (TUBITAK) is acknowledged for granting International Post Doctoral Research Fellowship Program in the framework of TUBITAK-BIDEB 2219 grant. The author thanks to the BAMM LABs for hosting our experiments, especially to Utkan
Demirci, Umut Atakan Gürkan for technical discussions.

\section{References}

[1] Slaughter, B.V.; Khurshid, S.S.; Fisher, O.Z.; Khademhosseini, A.; Peppas, N.A. Hydrogels in regenerative medicine. Advanced Materials, 2009; 21, 3307-3329.

[2] Wheeldon, I; Farhadi, A; Bick, A.G; Jabbari, E.; Khademhosseini, A. Nanoscale tissue engineering: spatial control over cell-materials interactions. Nanotechnology. 2011; 22, 212001.

[3] Kai, D.; Prabhakaran, M.P.; Stahl, B.; Eblenkamp, M.; Wintermantel, E.; Ramakrishna, S. Mechanical properties and in vitro behavior of nanofiber-hydrogel composites for tissue engineering applications. Nanotechnology. 2012; 23, 095705.

[4] Buyanov, A.; Gofman, I.; Revel'skaya, L.; Khripunov, A.; Tkachenko, A. Anisotropic swelling and mechanical behavior of composite bacterial cellulose-poly (acrylamide or acrylamide-sodium acrylate) hydrogels. Journal of the Mechanical Behavior of Biomedical Materials. 2010; 3, 102-11.

[5] Huang, T.; Xu, H.; Jiao, K.; Zhu, L.; Brown, H.R.; Wang, H. A novel hydrogel with high mechanical strength: a macromolecular microsphere composite hydrogel. Advanced Materials. 2007; 19, 1622-1626.

[6] Wu, Y.; Zhou, Z.; Fan, Q.; Chen, L.; Zhu, M. Facile insitu fabrication of novel organic nanoparticle hydrogels with excellent mechanical properties. Journal of Materials Chemistry. 2009; 19, 7340-7346.

[7] Stefanescu EA, Stefanescu C, Daly WH, Schmidt G, Negulescu II. Hybrid polymer-clay nanocomposites: a mechanical study on gels and multilayered films. Polymer. 2008;49:3785-94.

[8] Tong, X.; Zheng, J.; Lu, Y.; Zhang, Z.; Cheng, H. Swelling and mechanical behaviors of carbon nanotube/poly (vinyl alcohol) hybrid hydrogels. Materials Letters. 2007; 61, 1704-1706.

[9] Gong, J.P.; Katsuyama, Y.; Kurokawa, T.; Osada, Y. Double-Network Hydrogels with Extremely High Mechanical Strength. Advanced Materials. 2003; 15, 11551158.

[10] Lee, W.F.; Chen, Y.J. Studies on preparation and swelling properties of the $\mathrm{N}$ isopropylacrylamide/chitosan semi-IPN and IPN 
hydrogels. Journal of Applied Polymer Science. 2001; 82, 2487-2496.

[11] Peppas, N.A. Hydrogels in medicine and pharmacy: CRC press Boca Raton, FL; 1988.

[12] Brannon-Peppas, L; Peppas, N.A. Equilibrium swelling behavior of $\mathrm{pH}$-sensitive hydrogels. Chemical Engineering Science. 1991; 46, 715-722.

[13] Chen, R.R.; Mooney, D.J. Polymeric growth factor delivery strategies for tissue engineering. Pharmaceutical Research. 2003; 20, 1103-1112.

[14] Am Ende, M.; Hariharan, D.; Peppas, N. Factors influencing drug and protein transport and release from ionic hydrogels. Reactive Polymers. 1995; 25, 127-137.

[15] Brannon-Peppas, L.; Peppas, N.A. Solute and penetrant diffusion in swellable polymers. IX. The mechanisms of drug release from $\mathrm{pH}$-sensitive swellingcontrolled systems. Journal of Controlled Release. 1989; 8, 267-274.

[16] Collins, M.C.; Ramirez, W.F. Transport through polymeric membranes. The Journal of Physical Chemistry. 1979; 83, 2294-2301.

[17] Sannino, A.; Demitri, C.; Madaghiele, M. Biodegradable cellulose-based hydrogels: design and applications. Materials 2009; 2, 353-373.

[18] Tanaka, T. Kinetics of phase transition in polymer gels. Physica A: Statistical Mechanics and its Applications. 1986; 140, 261-268.

[19] Mao, L.; Hu, Y.; Piao, Y.; Chen, X.; Xian, W.; Piao, D. Structure and character of artificial muscle model constructed from fibrous hydrogel. Current Applied Physics. 2005; 5, 426-428.

[20] Peppas, N.A. Hydrogels and drug delivery. Current Opinion in Colloid \& Interface Science. 1997 2, 531-537.

[21] Qiu, Y.; Park, K. Environment-sensitive hydrogels for drug delivery. Advanced Drug Delivery Reviews. 2001; 53, 321-339.

[22] Richter, A.; Howitz, S.; Kuckling, D.; Arndt, K.F. Influence of volume phase transition phenomena on the behavior of hydrogel-based valves. Sensors and Actuators B: Chemical. 2004; 99, 451-458.

[23] Tan, G.; Chen, R.; Ning, C.; Zhang, L.; Ruan, X.; Liao, $\mathrm{J}$. Effects of argon plasma treatment on surface characteristic of photopolymerization PEGDA-HEMA hydrogels. Journal of Applied Polymer Science. 2012; 124, 459-465.

[24] Hong, Y.; Song, H.; Gong, Y.; Mao, Z.; Gao, C.; Shen, J. Covalently crosslinked chitosan hydrogel: properties of in vitro degradation and chondrocyte encapsulation. Acta Biomaterialia. 2007; 3, 23-31.

[25] Lee, K.Y.; Mooney, D.J. Hydrogels for tissue engineering. Chemical Reviews. 2001; 101, 1869-1880.

[26] Nicodemus, G.D.; Bryant, S.J. Cell encapsulation in biodegradable hydrogels for tissue engineering applications. Tissue Engineering Part B: Reviews. 2008; $14,149-165$.

[27] Peppas, N.A.; Hilt, J.Z.; Khademhosseini, A.; Langer, R. Hydrogels in biology and medicine: from molecular principles to bionanotechnology. Advanced Materials. 2006; 18, 1345-1360.

[28] Schweikl, H.; Spagnuolo, G.; Schmalz, G. Genetic and cellular toxicology of dental resin monomers. Journal of Dental Research. 2006; 85, 870-877.

[29] Sokullu-Urkac, E.; Oztarhan, A.; Tihminlioglu, F.; Nikolaev, A.; Brown, I. Oxidation Behavior of C-and AuIon-Implanted Biodegradable Polymers. IEEE Transactions on Plasma Science. 2012; 40, 863-869.

[30] Temenoff, J.S.; Park, H.; Jabbari, E.; Conway, D.E.; Sheffield, T.L.; Ambrose, C.G. Thermally cross-linked oligo (poly (ethylene glycol) fumarate) hydrogels support osteogenic differentiation of encapsulated marrow stromal cells in vitro. Biomacromolecules 2004; 5, 5-10.

[31] Temenoff, J.S.; Park, H.; Jabbari, E.; Sheffield, T.L.; LeBaron, R.G.; Ambrose, C.G. In vitro osteogenic differentiation of marrow stromal cells encapsulated in biodegradable hydrogels. Journal of Biomedical Materials Research Part A. 2004; 70, 235-244.

[32] Sokullu-Urkac, E.; Oztarhan, A.; Tihminlioglu, F.; Kaya, N.; Ila, D.; Muntele, C. Thermal characterization of $\mathrm{Ag}$ and $\mathrm{Ag}+\mathrm{N}$ ion implanted ultra-high molecular weight polyethylene (UHMWPE). Nuclear Instruments and Methods in Physics Research Section B: Beam Interactions with Materials and Atoms. 2007; 261, 699703. 